\title{
Magnetism of Higher Borides
}

\begin{abstract}
T. MORI
National Institute for Materials Science, Nanoscale Materials Center

Namiki 1-1, Tsukuba, 305-0044, Japan*

Magnetism of borides which contain the $\mathrm{B}_{12}$ icosahedra as a structural building block are attracting increasing interest since they exhibit unexpectedly strong magnetic interactions, despite being magnetically dilute $f$-electron insulators. The magnetic behavior among the different compounds has also been found to be diverse. The $f$-electron dependence of magnetic $\mathrm{B}_{12}$ icosahedra borides is compared, and found to be different from conventional mechanisms. The $\mathrm{TbB}_{25}$ system is also investigated further and the transition is assigned to a typical antiferromagnetic transition.
\end{abstract}

PACS numbers: 81.05.Je, 75.50.Ee

\section{Introduction}

The magnetism of rare earth borides like tetraborides $\mathrm{REB}_{4}$, hexaborides $\mathrm{REB}_{6}$, and dodecaborides $\mathrm{REB}_{12}$ has attracted a lot of attention over the years [1-6], with recent interesting discoveries still being made such as the complex magnetic structure revealed in $\mathrm{REB}_{12}$ [7]. These compounds are all good metals in the case of trivalent rare earth elements, and their magnetic coupling has basically been described by the Ruderman-Kittel-Kasuya-Yosida (RKKY) mechanism with possible secondary effects from the dipole-dipole interaction. As an emerging novel phenomenon, it has been found that borides which contain the $\mathrm{B}_{12}$ icosahedra as a structural building block [8] can exhibit unexpectedly strong magnetic interactions, although they are relatively magnetically dilute, $f$-electron insulators. For example, $T_{\mathrm{N}}=18 \mathrm{~K}$ for $\mathrm{TbB}_{50}[10]$ and the peak temperature in the zero field cooled (ZFC) susceptibility of $\mathrm{HoB}_{17} \mathrm{CN}$ is $T_{*}=29 \mathrm{~K}$ [9]. A wide variation of the magnetic behavior has also been observed, ranging from the one-dimensional dimer-like antiferromagnetic transition in $\mathrm{REB}_{50}$ and $\mathrm{REB}_{44} \mathrm{Si}_{2}$ $[9,11,12]$, two-dimensional spin-glass behavior in $\mathrm{REB}_{17} \mathrm{CN}, \mathrm{REB}_{22} \mathrm{C}_{2} \mathrm{~N}$, and $\mathrm{REB}_{28.5} \mathrm{C}_{4}[10,13,14]$ to three-dimensional long-range order in $\mathrm{GdB}_{18} \mathrm{Si}_{5}$ [15].

We analyze the $f$-electron dependence of some of the magnetic higher borides which contain the $\mathrm{B}_{12}$ icosahedra. Furthermore, a low temperature

* presently visiting Max Planck Institute for Chemical Physics of Solids, Nothnitzer Str. 40, 01187 Dresden, Germany 
antiferromagnetic-like transition was previously reported in $\mathrm{TbB}_{25}$ [16], and we investigate the nature of this transition in more detail.

\section{Experimental}

In regards to synthesis, $\mathrm{TbB}_{25}$ was synthesized by the borothermal reduction of terbium oxide under vacuum; $\mathrm{Tb}_{2} \mathrm{O}_{3}+53 \mathrm{~B} \rightarrow 2 \mathrm{TbB}_{25}+3 \mathrm{BO}$, as described previously [16]. The reaction was performed under vacuum in a $\mathrm{BN}$ crucible surrounded by an inductively heated composite susceptor at $1900^{\circ} \mathrm{C}$. Characterization was done by powder X-ray diffractometry and chemical analysis (AES-ICP). Magnetic properties were measured with a SQUID magnetometer (Quantum Design MPMS).

\section{Results and discussion}

First of all, the magnetic coupling mechanism in the insulating higher borides is considered. The characteristic temperatures of the magnetism for some $\mathrm{B}_{12}$ icosahedra containing rare earth borides are plotted in Fig. 1. The expected dependences in the case of the conventional $f$-electron mechanisms, RKKY mechanism and dipole-dipole interaction are also shown with the value for Er arbitrarily set as $4.5 \mathrm{~K}$, which is the characteristic temperature of $\mathrm{ErB}_{28.5} \mathrm{C}_{4}$.

The RKKY interaction scales with the de Gennes factor [17]

$$
\text { Int }_{\mathrm{RKKY}} \propto\left(g_{J}-1\right)^{2} J(J+1)
$$

where $g_{J}$ is the Lande factor and $J$ - the total angular momentum. The dipoledipole interaction can approximately be expressed by [18]

$$
\operatorname{Int}_{\text {dipole }} \propto g_{J}^{2} \mu_{\mathrm{B}}^{2} J(J+1) .
$$

As shown in Fig. 1, we find that the $f$-electron dependences of the characteristic temperatures of the magnetic behavior observed for the higher borides do not simply follow either mechanism. This is also underlined by the fact that the $\mathrm{Tb}$ phase has the highest $T_{\mathrm{N}}$ for $\mathrm{REB}_{25}$ [16], while $\mathrm{Gd}$ has the highest for $\mathrm{REB}_{18} \mathrm{Si}_{5}[15]$.

As noted in the introduction, the magnetic behavior in higher borides manifests in a wide variety of forms. The reported transition at $2.1 \mathrm{~K}$ in $\mathrm{TbB}_{25}$ is investigated in more detail by varying the magnetic field.

The magnetic field dependence of $\chi$ of $\mathrm{TbB}_{25}$ is plotted in Fig. 2a. The transition temperature $T_{\mathrm{N}}$ shifts to lower temperatures as the magnetic field is increased. We determine $T_{\mathrm{N}}$ as the temperature, where $\mathrm{d} \chi / \mathrm{d} T=0$ for each magnetic field. If we assume a simple quadratic suppression of $T_{\mathrm{N}}$ due to magnetic fields as determined, for example, by Shapira and Foner [19]

$$
T=T_{\mathrm{N}}\left(1-\left(H / H_{\mathrm{C}}\right)^{2}\right),
$$

we can obtain a good fitting curve as shown in Fig. 2b. Parameters are determined as $T_{\mathrm{N}}=2.1 \mathrm{~K}$ and $H_{\mathrm{C}}=5.1 \mathrm{kOe}$. This determined value of $H_{\mathrm{C}}$ is within the 


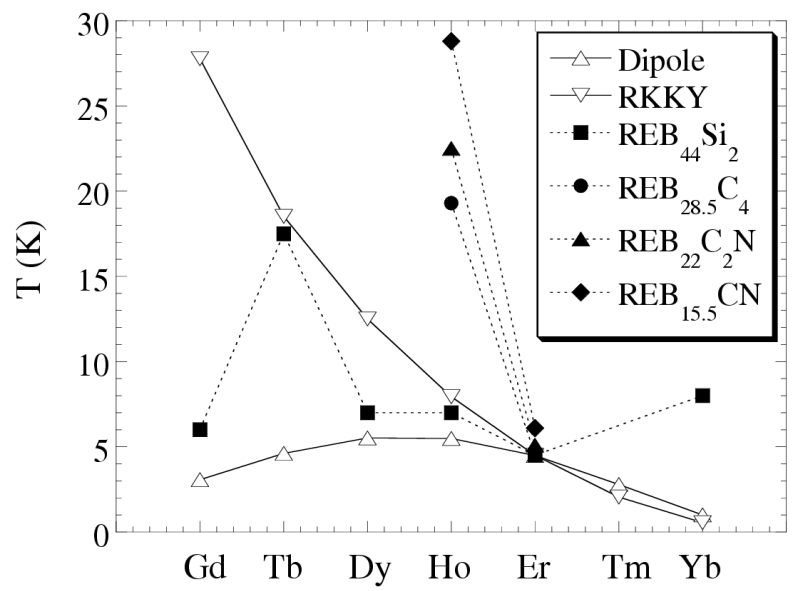

Fig. 1. Rare earth dependence of the characteristic temperatures of the magnetism of rare earth higher borides. $T_{\mathrm{N}}$ for $\mathrm{REB}_{44} \mathrm{Si}_{2}[11,12]$ and the peak temperature $T_{*}$ of $\chi_{\mathrm{ZFC}}$ for $\mathrm{REB}_{28.5} \mathrm{C}_{4}, \mathrm{REB}_{22} \mathrm{C}_{2} \mathrm{~N}$, and $\mathrm{REB}_{15.5} \mathrm{CN}[13,14,10]$ are plotted. Theoretical values for the RKKY interaction and dipole-dipole mechanism are also plotted after normalizing at an arbitrary value of $4.5 \mathrm{~K}$ for erbium for visual clarity.
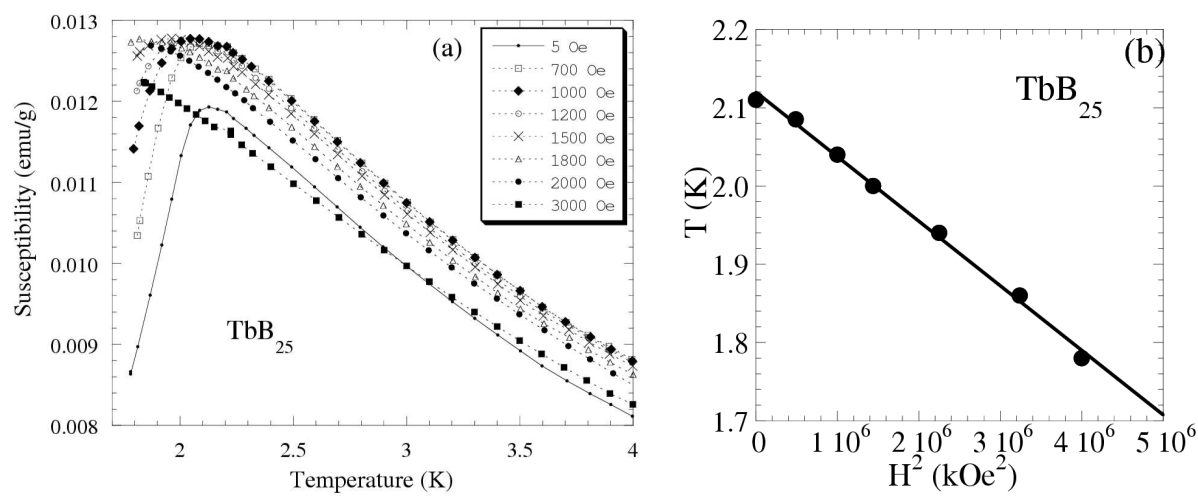

Fig. 2. (a) Magnetic field dependence of the magnetic susceptibility for $\mathrm{TbB}_{25}$. (b) The fit to Eq. (4), $T=T_{\mathrm{N}}\left(1-\left(H / H_{\mathrm{C}}\right)^{2}\right)$, yields parameters of $T_{\mathrm{N}}=2.1 \mathrm{~K}$ and $H_{\mathrm{C}}=5.1 \mathrm{kOe}$. magnitude of order which is expected for local moment antiferromagnets: $H_{\mathrm{C}} \sim$ $k_{\mathrm{B}} T_{\mathrm{N}} / \mu_{\mathrm{B}}=14 \mathrm{kOe}$.

Hysteresis is not observed and it is indicated that the transition in $\mathrm{TbB}_{25}$ is a typical antiferromagnetic transition. 


\section{References}

[1] B.T. Matthias, T.H. Geballe, K. Andres, E. Corenzwit, G.W. Hull, J.P. Maita, Science 159, 530 (1968).

[2] Z. Fisk, M.B. Maple, D.C. Johnston, L.D. Woolf, Solid State Commun. 39, 1189 (1981).

[3] J. Etourneau, J. Less Common Met. 110, 267 (1985).

[4] Y. Paderno, N. Shitsevalova, I. Batko, K. Flahbart, H. Misiorek, J. Mucha, A. Jezowski, J. Alloys Comp. 219, 215, 95 (19.)

[5] D. Gignoux, D. Schmitt, Handbook of Magnetic Materials, Vol. 10, Ed. K.H.J. Buschow, North-Holland, Amsterdam 1997, p. 239.

[6] S. Gabani, I. Bat'ko, K. Flachbart, T. Herrmannsdoerfer, R. Koenig, Y. Paderno, N. Shitsevalova, J. Magn. Magn. Mater. 207, 131 (1999).

[7] K. Siemensmeyer, K. Flachbart, S. Gabani, S. Mat'as, Y. Paderno, N. Shitsevalova, J. Solid State Chem. 179, 2748 (2006).

[8] T. Mori, in: Handbook on the Physics and Chemistry of Rare Earth, Vol. 38, Eds. K.A. Gschneidner Jr., J.-C. Bunzli, V. Pecharsky, North-Holland, Amsterdam 2008, p. 105.

[9] T. Mori, T. Tanaka, J. Phys. Soc. Jpn. 68, 2033 (1999).

[10] T. Mori, F. Zhang, A. Leithe-Jasper, J. Solid State Chem. 177, 444 (2004).

[11] T. Mori, J. Appl. Phys. 95, 7204 (2004).

[12] T. Mori, Z. Kristallogr. 221, 464 (2006).

[13] T. Mori, A. Leithe-Jasper, Phys. Rev. B 66, 214419 (2002).

[14] T. Mori, H. Mamiya, Phys. Rev. B 68, 214422 (2003).

[15] T. Mori, F. Zhang, J. Phys., Condens. Matter 14, 11831 (2002).

[16] T. Mori, F. Zhang, T. Tanaka, J. Phys., Condens. Matter 13, L423 (2001).

[17] M. A. Ruderman, C. Kittel, Phys. Rev. 96, 99 (1954); T. Kasuya, Prog. Theor. Phys. 16, 45 (1956); K. Yosida, Phys. Rev. 106, 893 (1957).

[18] J. Kanamori, Jisei, Baifukan, Tokyo 1969, p. 107 (in Japanese).

[19] Y. Shapira, S. Foner, Phys. Rev. B 1, 3083 (1970). 